\title{
Lyapunov-Based Control for Switched Power Converters *
}

\author{
Seth R. Sanders \\ Department of Electrical Engineering \\ and Computer Sciences \\ University of California, Berkeley
}

\author{
George C. Verghese \\ Laboratory for Electromagnetic \\ and Electronic Systems \\ Massachusetts Institute of Technology
}

\begin{abstract}
Beginning with fundamental properties such as passivity or incremental passivity of the network elements comprising a switched power converter, the nominal open-loop operation of a broad class of such converters is shown to be stable in the large via a Lyapunov argument. The obtained Lyapunov function is then shown to be useful for designing globally stabilizing controls that include adaptive schemes for han. dling uncertain nominal parameters. Numerical simulations illustrate the application of this control approach in DC-DC converters.
\end{abstract}

\section{Introduction}

Most control schemes for power electronic circuits in present use are obtained by linearizing a nonlinear model about a nominal operating point or trajectory. Large signal transients that occur at power up or overload recovery are handled in an ad hoc manner. Designers analyze each circuit individually to prescribe a scheme to accomodate a designated set of large signal trausients. This paper addresses the issue of how one might do better. In particular, the paper develops a methodology for designing control laws for fast-switching converters that result in globally stable behavior, robustness against parametric uncertainty, and satisfactory transient response. The main approach in this paper is based on the use of Lyapunov functions.

There has been considerable previous work along these lines. The paper of Erickson et al. [11] develops a large-signal averaged model for switched converters and points out the hazards of control designs based upon small-signal, linearized models. The previous work on large signal control schemes can be divided roughly into two groups. One is based on so called "switching-law" controls where the position of a controlled switch is directly commanded as a function of the instantaneous values of the circuit variables. Examples of these are the sliding mode control schemes of $[1,2,3,4,5,6,7,21]$ (which include current-mode control) and the bang-bang control schemes of $[9,10,15]$. The second approach relies on the state-space averaged model for the converter of interest. Representative schemes are those in $[12,13,14,22]$. References

-The first author has been partially supported by an IBM fellowship. The second author has been supported by the MIT/Industry Power Electronics Collegium and by the Air Force Office of Scientific Research under Grant AFOSR-88-0032
[12] and [14] also consider coutrol design using Lyapunov fuuctions.

The paper is organized as follows. Section 2 develops fundamental stability properties for a broad class of switching converters. In particular, we derive the form of a Lyapunov function that illustrates that each member of this class is open-loop stable. In Section 3, the Lyapunov-based control approach is introduced. This section includes a simple example to demonstrate the method, an outline of the general approach, and an illustration of how an adaptation scheme can be incorporated to handle uncertainties in the nominal operating point.

\section{Open-Loop Stability of Switch- ing Converters}

In this section, switching converter systems (understood to include source and load) that consist of an intercounection of ideal DC sources, ideal switches, incrementally passive resistors, and passive linear reactive elements are consiclered. Multiport circuit elements are included in the development here. This class of switching converters is shown to be stable by exhibiting a Lyapunov function that corresponds to the energy in the increment with respect to an arbitrary, nominal state trajectory. The argument is extencled to include nonlinear reactive elements that are strictly relatively passive in the case where the switching frernency becomes infinite, and stability with respect to an equilibrium point is considered. Essential background on network theoretic issues for the development in this section is contained in Appendix A. (Also, see $[16,17,18]$.)

\subsection{Switching Converter Stability Under Finite Switching Frequency}

Let the switching converter be composed of icleal DC. sources, ideal switches, incrementally passive resistors, and linear passive reactive elements. A diode may be considered as either an ideal switch or as an incrementally passive resistor. IVe suppose the switches are operated in accord with a given arbitrary switching pattern and suppose that we are given a nominal solution corresponding to the given switching pattern.

For each branch of the network, denote the nominal trajectory by $\{\tilde{v}(t), \tilde{i}(t)\}$, and form the (not necessarily small) increments with respect to the nominal trajectory for each 
network branch, i.e.

$$
\begin{aligned}
& \delta i(t)=i(t)-\tilde{i}(t) \\
& \delta v(t)=v(t)-\tilde{v}(t)
\end{aligned}
$$

By applying Tellegen's theorem to the increments in all the network branches when the circuit is in any one of its topologies, we obtain

$$
\begin{aligned}
0= & \sum_{D C \text { sources }} \delta i \delta v+\sum_{\text {Switches }} \delta i \delta v+\sum_{\text {Res. }} \delta i \delta v+ \\
& \sum_{\text {Ind. }} \delta i \delta v+\sum_{\text {Cap. }} \delta i \delta v
\end{aligned}
$$

The summation involving DC sources is always zero since the increment in either voltage or current of each term is necessarily zero. The terms involving switches also add zero contribution to the sum in (2) for the same reason. The third summation on the right-hand side of $(2)$ is always nonuegative since each term is individually positive or zero as a result of the incremental passivity of the resistors. In fact, this summation can be thought of as the dissipated power in the increment with respect to the given nominal trajectory. The remaining two summations represent the time rate of change of the stored energy in the increment. Combining these facts, we can write:

$$
\frac{d}{d t} \mathrm{~V}(\delta x)=\sum_{\text {lnd. }} \delta i \delta v+\sum_{\text {Cap. }} \delta i \delta v=-\sum_{\text {Res. }} \delta i \delta v
$$

where

$$
\begin{aligned}
\mathrm{V}(\delta x)= & \sum_{\text {Ind. }}(1 / 2)\left(\delta i_{k}\right)^{*} L_{k}\left(\delta i_{k}\right)+ \\
& \sum_{\text {Cap. }}(1 / 2)\left(\delta v_{k}\right)^{*} C_{k}\left(\delta v_{k}\right) .
\end{aligned}
$$

(Note that the superscript - indicates transpose.) Because of the assumption on passivity of the reactive elements, the quantity $\mathrm{V}(\delta \cdot x)$ which we shall from now on refer to by the suggestive name energy in the increment, is a positive definite quadratic function of the incremental state variables. Since equation (2) holds iclentically for any of the possible circuit topologies, the energy in the increment is a Lyapunov function for the dynamical system. In particular, we have

$$
\frac{d}{d t} \mathrm{~V}(\delta x)=-\sum_{\text {Reo. }} \delta v \delta i \leq 0
$$

In conclusion, the energy in the increment is a Lyapunov functiou for the given nominal trajectory, and we see that the nominal trajectory is stable in the large. Since the nominal trajectory selected above can be taken as any solution trajectory, this statement implies that any two solution trajectories do not diverge.

Typically, asymptotic stability in the large can be concluded as well since at least some parasitic loss is always associated with each energy storage element, i.e. series resistance with inductors and parallel leakage resistance with capacitors. An argument for asymptotic stability appears in [16] for circuits that have a DC equilibrium point, and consist of only two-terminal elements. In [16], lossiness is guaranteed to be associated with each state variable by excluding inductorcapacitor-voltage source loops and inductor-capacitor-current source cutsets. (In this case, we would require that all resistors be strictly incrementally passive.)

A special case of the abore result is when the switches are operated with a periodic switching pattern, and there exists a nominal periodic steady state solution. In this case, the result states that the given periodic steady state trajectory is stable in the large. This result is of particular interest for the case of a DC-DC converter operating with constant switching frequency. Note that this result holds up for DC-DC converters operating in the discontinuous conduction mode. This can be seen by redrawing the schematic for the DC-DC converter of interest with an ideal SPDT switch and incrementally passive resistive device (i.e. diode) replacing each transistor-diode pair. For example, we would redraw the up-down converter of Figure 2 as shown in Figure 1. The circuit of Figure 1 satisfies the conditions for its nominal periodic trajectory to be stable in the large, and it makes no difference whether or not the nominal trajectory contains a portion where the inductor current is identically zero.

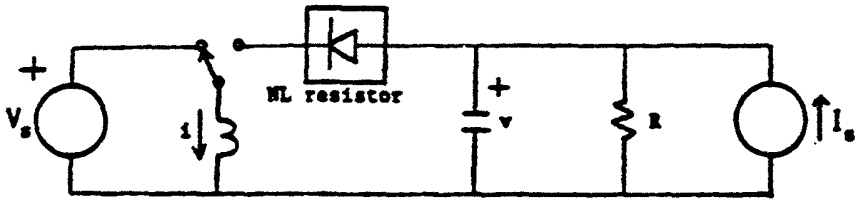

Figure 1: Up-Down Converter Redrawn to Ilustrate Stability in Case of Discontinuous Conducion

\subsection{Stability under Infinite Switching Fre- quency and Constant Duty Ratio}

The result given above can be extended to the case where the switching frequency becomes infinite. Infinite switching frequency actually corresponds to the state-space averaged model for a converter, and in this way an open-loop stability result can be obtained for state-space areraged models. The main difference from the case of finite switching frequency is that one needs to consicler the stability of an equilibrium point for an averaged model of a DC-DC converter rather than a limit cycle. In such a set-np, it is possible to include noulinear reactive elements as well as nonlinear resistive elements. This is of interest in power electronic circuits since noulinear reactive elements do occur in practice. The following theorem summarizes the result for infinite switching frequency.

Theoren 2.1 Suppose that a switching converter is constructed from ideal surtches, ideal DC sources, incrementally passive resistors, reactive elements that are strictly relatively passive, and that its averaged model has an equilibrium point, then the equilibrium is stable in the large.

This theorem is easily proven by demonstrating the existence of an appropriate averaged circuit model. See [23] for more details. We sball rely heavily on this result in the sequel where control laws based on state-space averaged models are developed. 


\section{Lyapunov-Based Control Design}

In this section, an approach to control of switching power converters based on the use of Lyapunov functions will be introduced. The main focus will be on control design based on the state-space averaged model for a given switching converter. The converters of interest are those that satisfy the conditions guaranteeing that nominal state trajectories are globally stable under open-loop operation, specifically converters constructed from incrementally passive resistors, ideal sources, ideal switches, and passive linear reactive elements. One particular choice of Lyapunov function for control de sign purposes that will be of interest is the energy in the increment.

We shall begin by illustrating the Lyapunov-based control method with an application to an up-down converter. Then, we shall demonstrate how such a control design can be obtained in a more general way. There is typically some freedom in the cboice of Lyapunov function for the control design, but we shall exhibit some particular advantages of using the energy in the increment. Finally, we show how an adaptation scheme can be incorporated to handle parametric uncertainty. Generalizations to converters containing nonlinear circuit elements, to converters that handle time-varying input-output waveforms, and to converters operating in the discontinuous conduction mode are given in the thesis [23]. A method (dual to the control design approach) for designing state observers is also considered in [23].

\subsection{Example: Up-Down Converter}

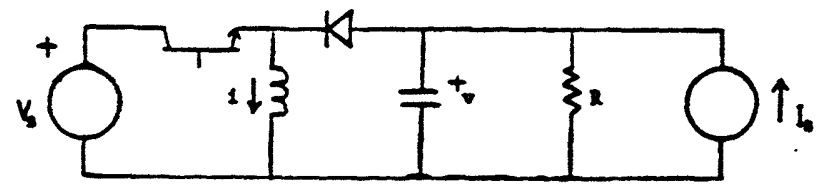

Figure 2: Up-Down Converter

Consider the up-down converter of Figure 2 which has a statespace areraged model of the form

$$
x^{\prime}=A x+(B x+b) d,
$$

where the two-component state $x$ consists of the deviation of the inductor current from its nominal value $\left(x_{1}=i-i_{n}\right)$ and the deviation of the capacitor voltage from its nominal $\left(x_{2}=v-v_{n}\right)$, and where the input $d$ is the deviation in the duty ratio from its nominal value $\left(d=d_{t}-d_{n}\right)$. (Note that $d_{t}$ indicates the total duty ratio here.) The parameter values listed below were selected for operation at a switching frequeucy of $50 \mathrm{KHz}$.

$$
\begin{aligned}
C & =5.4 \mu \mathrm{F} \\
L & =0.18 \mathrm{mH} \\
R & =\infty \\
d_{n} & =3 / 8 \\
V_{0} & =15 \text { volts }
\end{aligned}
$$

$$
\begin{aligned}
& I_{0}=2 \text { amps } \\
& v_{n}=-9 \text { volts } \\
& i_{n}=3.2 \mathrm{amps}
\end{aligned}
$$

The relevant matrices of the system are as follows:

$$
\begin{aligned}
& A=\left[\begin{array}{cc}
0 & \left(1-d_{n}\right) / L \\
-\left(1-d_{n}\right) / C & 0 \\
0 & -1 / L \\
1 / C & 0 \\
\left(V_{0}-v_{n}\right) / L \\
i_{n} / C \\
L
\end{array}\right] \\
& {\left[\begin{array}{cc}
L & 0 \\
0 & C
\end{array}\right]}
\end{aligned}
$$

The result on open-loop stability in Section 2 guarantees that the energy in the increment is a Lyapunov function for openloop operation of this circuit. For the up-down converter, the energy in the increment takes the form

$$
\begin{gathered}
V=\frac{1}{2} L\left(i-i_{n}\right)^{2}+\frac{1}{2} C\left(v-v_{n}\right)^{2}, \\
V=\frac{1}{2} x^{*} Q x .
\end{gathered}
$$

Differentiating $V$ along the system trajectories, we obtain

$$
\begin{aligned}
\frac{d}{d t} V(x)= & \frac{1}{2} x^{*}\left(Q A+A^{*} Q\right) x+ \\
& \frac{1}{2}\left\{x^{*}\left(Q B+B^{*} Q\right) x+2 b^{*} Q x\right\} d .
\end{aligned}
$$

It turns out that $Q A+A^{*} Q=0$ for this example, winich verifies that the energy in the increment is a Lyapunov function for open-loop operation $(d=0)$. In this example, it is also true that $Q B+B^{*} Q=0$. These relationships hold because of the lossless nature of the example converter, i.e. the lack of resistive elements in the converter. Considering these relationships, (9) simplifies considerably to

$$
\frac{d}{d t} V(x)=\left(b^{*} Q x\right) d
$$

Many stabilizing control schemes can be obtained by inspection of (10). We shall consider the simple control law $d=-a b^{*} Q x$ with $\alpha$ real and positive, modified to haudle the duty ratio saturation constraint $-d_{n} \leq d \leq 1-d_{n}$, i.e.

$$
d= \begin{cases}-\alpha y, & -d_{n} \leq d \leq 1-d_{n} \\ -d_{n}, & -\alpha y<-d_{n} \\ 1-d_{n}, & -\alpha y>1-d_{n}\end{cases}
$$

where $y=b^{*} Q x$. Here, the variable $y$ takes the form

$$
\begin{aligned}
y & =\left(V-v_{n}\right)\left(i-i_{n}\right)+i_{n}\left(v-v_{n}\right) \\
& =(V-v)\left(i-i_{n}\right)+i\left(v-v_{n}^{*}\right) .
\end{aligned}
$$

Note that the only dependence on circuit parameters is on the nominal values of the inductor current, the capacitor voltage, and the source voltage. This property is shared by analagous control schemes based on the energy in the increment for 
many $\alpha$ her switching converters, as will be discussed in Subsection 3.3. The dependence on nominal values of circuit variables is of crucial importance, and this issue is addressed in Subsection 3.4. There, a method for adaptively estimatiug these ralues is developed.

To irrestigate the closed-loop behavior, we examine the derivative of the Lyapunov function $V(x)$ along the closedloop system trajectories:

$$
\begin{aligned}
\frac{d}{d t} V(x) & =y d \\
& = \begin{cases}-\alpha y^{2}, & -d_{n} \leq d \leq 1-d_{n} . \\
-d_{n} y, & -\alpha y<-d_{n} \\
\left(1-d_{n}\right) y, & -\alpha y>1-d_{n}\end{cases}
\end{aligned}
$$

In the saturated regions (the second and third lines of (13)), the time derivative of $V(x)$ is strictly negative since either $V^{\prime}<-a d_{n}^{2}$ or $V^{\prime}<-\alpha\left(1-d_{n}\right)^{2}$. As a result, state trajectories quickly enter the unsaturated region. In the unsaturated region (the first line in (13)), $V(x)$ is strictly decreasing if $y \neq 0$, and asymptotic stability can be concluded by LaSalle's theorem since $y \equiv 0$ is not a system trajectory unless $x \equiv 0$. To see this, note that $y \equiv 0$ implies $d \equiv 0$ and the following:

$$
\begin{aligned}
b^{*} Q x & =0 \\
b^{*} Q A x & =0,
\end{aligned}
$$

with the last line in (14) obtained by noting that $y^{\prime}=0$. The existence of a nonzero solution $x$ to (14) is equivalent to the statement that the pair $\left\{b^{*} Q, A\right\}$ is unobservable. However, this pair is observable in this example, and therefore there are no system trajectories that do not converge to the origin.

In this example, we have not considered the effect of lossiness dze to parasitic and/or load resistances. The effect of such passive resistances would only enhance our stability result, by causing additional nonpositive terms of the form $-x^{*} R_{x}$ (with $R$ positive semi-definite) to be added to the terms ou the right-hand sides of (13).

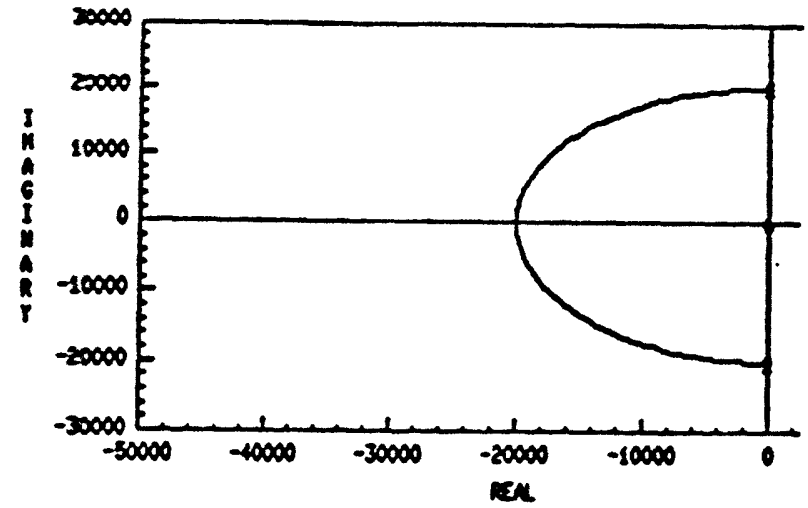

Figure 3: Root-Locus for Linearized Closed-Loop Control System

Asymptotically, the decay of the Lyapunov function $V(x)$ is conirolled by the eigenvalues of the small signal model obtained by linearizing the closed-loop system about $x=0$. In this example, there is some freedom in placing the eigenvalues of the linearized closed-loop system by choice of the gain $\alpha$. A root locus of the closed-loop eigenvalues of the small-signal model is shown in Figure 3. To ninimize the maximum of the real parts of the eigenvalues, for example, the gain should be selected so that the two eigenvalues coincide on the real axis at $-20.05 \mathrm{Krad} / \mathrm{sec}$. An easy calculation indicates that the value of the gain required to obtain this eigenvalue placement is approximately $\alpha=.00785$. In the remainder of the discussion of this example, a value of the gain of $\alpha=.008$ will be used. The resulting closed-loop eigenvalues are at about $-16.7 \mathrm{Krad} / \mathrm{sec}$ and $-24 \mathrm{Krad} / \mathrm{sec}$. Note that in this example the dynamical behavior of the small signal closedloop dynamics is limited by the natural resonant frequency $\left(1-d_{n}\right) / \sqrt{L C}$ of the open-loop state-space areraged system Since the bandwidth of the closed-loop dynamics is usually designed to be approximately an order of magnitude below the switching frequency, and since this is also a typical resonant frequency of the open-loop dynamics for a reasonably designed converter, the preceding limit on attainable closedloop bandwidth is acceptable.

We expect the closed-loop system to be very well behaved, and this is confirmed by the digital computer simulation shown in Figure 4. In the following subsection, we present
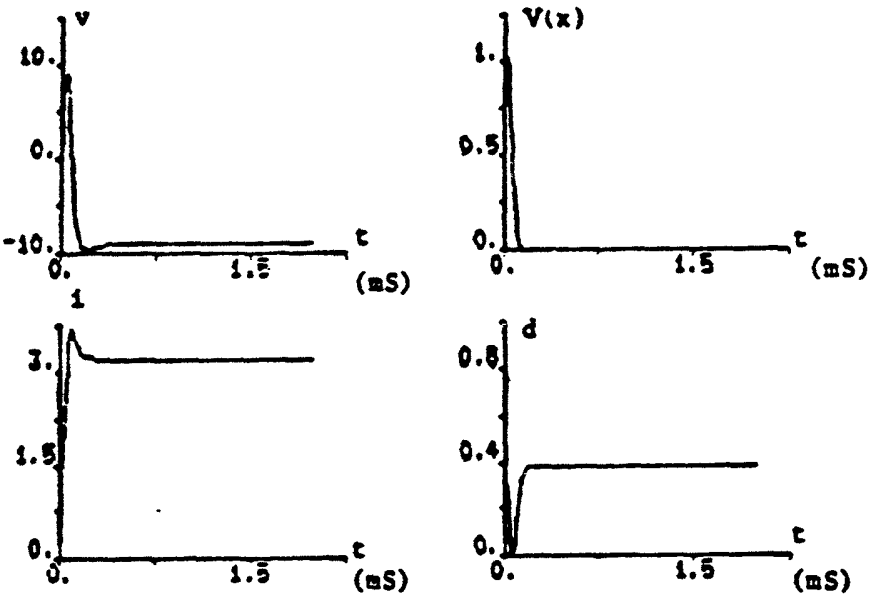

Figure 4: Digital Simulation of Up-Down Converter uuder Nonlinear Feedback Control Scheme

a derivation of a class of control schemes to which the above example belongs.

\subsection{A Basic Approach to Lyapunov-Based Control Design}

In this subsection, we show how to derive a class of control laws for a switching converter model of the form (6), to which the example (11) belongs. Note that the open-loop stability of the system (6) is crucial for this approach, and hence we shall restrict attention to switching converters that satisfy the conditions guaranteeing stability under nominal duty ratio operation. A basic first step in this approach, as illustrated above, is the specification of a Lyapunov function for openloop operation. The model $(6)$ is linear and time-invariant in the case of open-loop operation under a constant nominal duty ratio, i.e. $d=0$. Since the open-loop morlel is known a priori to be stable, it is generally possible to deternine 
a family of suitable quadratic Lyapunov functions. In fact, in the case where the matrix $A$ is asymptotically stable, it is possible to parametrize the family of such quadratic functions with the Lyapunov equation

$$
A^{*} Q_{1}+Q_{1} A=-P_{1} P_{1}^{*}
$$

where $\left\{P_{1}^{*}, A\right\}$ is an observable pair. The existence of a positive definite, symmetric solution $Q_{1}$ to (15) is guaranteed by the stability of the matrix $A$ and the observability of the pair $\left\{P_{i}^{*}, A\right\}[8]$. See [23] for a method of selecting a suitable natrix $Q_{1}$ for the case where the matrix $A$ has (simple) eigenvalues on the $j \omega$-axis.

Having deternined the form of a suitable matrix $Q_{1}$, it is straigh ; forward to specify a globally stabilizing control law for the model (6) of the form (11), but based on the Lyapunor function $V(x)=\frac{1}{2} x^{*} Q_{1} x$, as follows:

$$
d= \begin{cases}-\alpha y, & -d_{n} \leq-\alpha y \leq 1-d_{n} \\ -d_{n}, & -\alpha y<-d_{n} \\ 1-d_{n}, & -\alpha y>1-d_{n}\end{cases}
$$

where $y=(B x+b)^{*} Q_{1} x$. One particular choice for $Q_{1}$ is $Q$ where $Y(x)=\frac{1}{3} x^{*} Q . x$ is the energy in the increment, and it turus out that this choice leads to certain nice features, which are elaborated below.

\subsection{Advantages of the Use of the Energy in the Increment for Control Purposes}

As noted in the previous subsection, there is typically some freedow in the choice of the Lyapunov function that can be used in the control designs described there. Here, we outline three advantages obtained by using the energy in the incremeut as the Lyapunov function in these control schemes.

One advantage of the choice of the energy in the increment as the Lyapunov function for control design purposes arises in the computation of the variable $y=(B x+b)^{*} Q x$ which is used in these control schemes. In particular, one can always (indirectly) measure the vector $Q(B x+b)$. To see this, consider tze modification of $(6)$ where we multiply this equation on the left by the matrix $Q$, giving

$$
Q x^{\prime}=Q A x+Q(B x+b) d .
$$

Now the vector on the left-hand side of $(17)$ is composed of the time derivatives of the inductor fluxes and the time derivatives of the capacitor charges. The elements of this vector are necessarily inductor voltages and capacitor currents. The vector $Q(B x+b)$ is the amount by which this vector changes when the duty ratio steps from $-d_{n}$ to $1-d_{n}$, or equivalently, the amount this vector changes when the switch configuration is changed. In general, it is possible and feasible to determine the vector $Q(B x+b)$ during each cycle. To do this, for each inductor branch one would measure the voltage across the branch in each of the two switch configurations, and then form the difference of the two measurements. This difference constit utes the element of $Q(B x+b)$ corresponding to the particular inductor port. In the case of a capacitor, one would measure the current flowing into the capacitor in each of the two switch configurations, and form the difference of the two measured currents. This difference constitutes the element of $Q(B x+b)$ corresponding to the particular capacitor. By

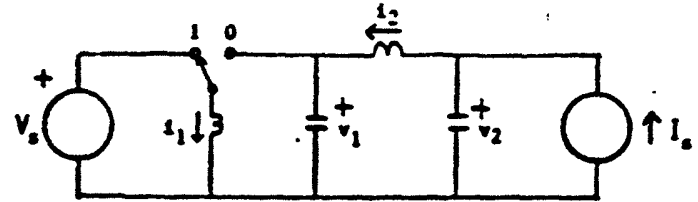

Figure 5: Up-Down Converter with Additional Filter Sections

performing the described measurement process, it is possible to obtain an accurate measurement of the vector $Q(B x+b)$. Consequently, one can compute the variable $y=x^{*} Q(B x+b)$ by forming the inner product of $x$ and $Q(B x+b)$. The only parametric dependence is therefore on the nominal state values required to determine $x$, the deviation in the states from their nominal values.

In certain cases, it is possible to further simplify the measurement of $Q(B x+b)$. For these cases, it is possible to directly measure the vector $Q(B x+b)$ by measuring certain branch voltages and branch currents in the circuit at one time instant. One such example is the up-down converter of Figure 2. For this example, the vector

$$
Q(B x+b)=\left[\begin{array}{c}
V_{a}-v \\
i
\end{array}\right]
$$

and these quantities can be directly measured on the converter circuit. Necessary and sufficient conditions for such a simple measurement of the vector $Q(B: x+b)$ are giveu in [23].

A second potential advantage of the choice $Q_{1}=Q$ in (16) is that it is possible to use a nearly linear version of this control algorithm by replacing $y=(B x+b)^{*} Q x$ in (16) with $y_{\text {lin }}=b^{*} Q x$, and still maintain global stability. (Of course, the saturation constraints are still in effect.) To see that global stability is maintained, consider the following Lyapunov analysis with $V(x)=\frac{1}{2} x^{*} Q x$ :

$$
\frac{d}{d t} V(x)=\frac{1}{2} x^{*}\left[(A+d B)^{*} Q+Q(A+d B)\right] x+\left(b^{*} Q x\right) d \text {. }
$$

Now the first term on the right-hand sicle of (19) is always nonpositive. This follows from the fact that the energy in the increment takes the form $\frac{1}{2} x^{*} Q x$ for any nomiual duty ratio, with the fixed matrix $Q$. The choice of the control in (16) (using $y_{\text {tin }}$ ) forces the second term on the right-haud side of (19) to be nonpositive. Global stability results from the nonpositivity of the right-hand side of (19). Hence, the choice of $Q_{1}=Q$ in (16) permits the use of a feedback control that requires only the computation of the linear variable $y_{\text {lin }}$.

A third advantage of the use of the energy in the increment as a Lyapunov function for control desigu is that a control law of the form (16) with $Q_{1}=Q$ can result in global stability of a more complex power system in which the original converter is embedded. In particular, if the converter is interconnected only with (relatively) passive circuit elements, the resulting interconnected system is always guaranteed to be stable. For example, if an additional section of output filter is addled to the up-down converter of Example 2, as shown in Figure 5, the control law designed for the original converter stalbilizes the modified circuit. 


\subsection{Adaptive Control Method to Handle Uncertain Nominal State Values}

This subsection considers a control design of the form (11) for the model (6), but in the case where the nominal state vector is unknown. The effect of this uncertainty is to replace the variable $y$ by

$$
y=(B x+b)^{*} Q\left(x-\delta x_{n}\right)
$$

where $\delta_{x_{n}}$ is the uncertainty in the nominal operating point, that is

$$
\delta x_{n}=\tilde{x}_{n}-x_{n}
$$

where $\tilde{I}_{n}$ is an estimate of the nominal operating point.

To implement the self-tuning scheme, we shall include as part of an augmented state vector, an estimate $\tilde{x}_{n}(t)$ of the constant nominal value of the state vector for the original plant. We can equivalently represent this estimate by its error, i.e. $\delta x_{n}(t)=\tilde{x}_{n}(t)-x_{n}$. The update law for $\delta x_{n}(t)$ is selected by considering the Lyapunov function

$$
V=\frac{1}{2} x^{*} Q x+\frac{1}{2}\left(\delta x_{n}\right)^{*} K\left(\delta x_{n}\right)
$$

where $K$ is a symmetric positive definite matrix and $Q$ is as previously specified. In particular, it is possible to stabilize the system by choosing the update law to be

$$
\frac{d}{d t}\left(\delta x_{n}\right)=-K^{-1} Q(B x+b)
$$

in conjunction with the control law (11). Note that $y$ can now be determined without any uncertainty arising from the unknown nominal state values since

$$
x-\delta x_{n}=\left(x_{t}-x_{n}\right)-\left(\tilde{x}_{n}-x_{n}\right)=x_{t}-\tilde{x}_{n},
$$

where $x_{l}$ is the actual full state value (which can be measured) while $\dot{I}_{n}$ is stored in the controller. Note that it is generally possible to obtain an accurate measure of $Q(B x+b)$ as discussed in Subsection 3.3.

Example: Estimation of Nominal Inductor Current in Simple Up-Down Converter In this example, we apply the adaptive control scheme to the secoud order up-down converter whose parameters are given in Section 3.1. We now assume, however, that the load is unknown but constant in the steady state. As a consequence, the nominal inductor current is also unkwown. This is the parameter that our self-tuning mechanism will estimate. In this example, it is assumed that the input voltage $V_{\text {a }}$ is known (i.e. measured), the nominal output voltage $v_{n}$ is defined by the regulation problem, and the nominal duty ratio $d_{n}$ is known. (The nominal duty ratio can usually be determined from $V_{0}$ and $v_{n}$.) We work with the augmented model

$$
\begin{gathered}
{\left[\begin{array}{c}
i^{\prime} \\
v^{\prime} \\
\left(\delta i_{n} y^{\prime}\right.
\end{array}\right]=\left[\begin{array}{ccc}
0 & \left(1-d_{n}\right) / L & 0 \\
-\left(1-d_{n}\right) / C & 0 & 0 \\
0 & 0 & 0
\end{array}\right]\left[\begin{array}{c}
i \\
v \\
\left(\delta i_{n}\right)
\end{array}\right]+} \\
\quad\left[\begin{array}{c}
\left(V-v_{t}\right) / L \\
i_{t} / C \\
-k^{-1}\left(V_{s}-v_{t}\right)
\end{array}\right] d \\
y=\left(V_{s}-v_{t}\right)\left\{i-\left(\delta i_{n}\right)\right\}+i_{t}\left(v-v_{n}\right)
\end{gathered}
$$

Note that in this model, the quantities without subscripts are deviations from nominal, the quantities with subscript $t$ are total variables that can be measured, and the quantities with subscript $n$ are nominal variables. We only attempt to estimate the nominal inductor current since the other nominal state variable (the capacitor voltage) is known. The output $y$ of this model can be deternined exactly since $i-\left(\delta i_{11}\right)$ is precisely $i_{t}-i_{n}$, i.e. the difference between the actual inductor current and the present estimate of the nominal value of this current. The control design can be completed by specifying $k>0$ and a feedback gain $\alpha$. These parameters may be selected by considering the small signal behavior. For example, with a nominal load current of 2 amps, the eigenvalues of the small signal linearized model can be placed at $-7.713 \pm j 12.9 \mathrm{Krad} / \mathrm{sec}$ and $-11.36 \mathrm{Krad} / \mathrm{sec}$ by select. ing $k=2778$ and the unsaturated gain $\alpha=.004$. Other parameter choices can result in still faster small signal behavior. A numerical simulation of a start-up transient using these parameters is shown in Figure 6. Note that the initial condition for the estimate of the nominal inductor was taken as zero.
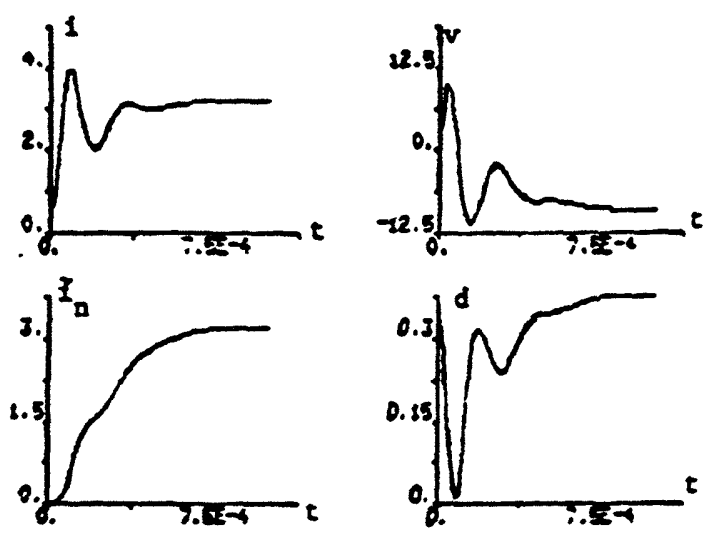

Figure 6: Start-Up Trausient in Second Order Couverter Using Adaptive Control Scheme

More complex examples that require estimation of more than one nominal state are considered in [23].

\section{Summarizing Remarks}

The Lyapunov-based control described in this paper is evidently a promising approach to the control of switched-mode power converters. The method can be exteuderl to couverters that handle time-varying input-output waveforms, see the discussion in [23]. In applications in distributed power supply environments, this type of control may prove very useful since it may become necessary to stabilize arbitrary interconnections of converters and loads. The method also lends itself (23) to the design of state observers as outlined in [23]. 


\section{A Passivity, Incremental Passivity, and Relative Passivity}

In order to state the following definitions in a relatively general war, we assume the input-output vector pair of an $n$-port to be a hybrid pair. That is, the input $u(t)$ and output $y(t)$ of an n-pert are $n$-component vectors whose elements represent port voltages or currents. The components of $y(t)$ are complementary to those of $u(t)$, and oriented such that $u(t)^{*} y(t)$ is the instantaneous power entering the network at its ports. In the following, the networks of interest are assumed to be time-invariant unless otherwise noted.

Passivity The definition of passivity presented in Wyatt et. al. [17], Wyatt [19], and in Hasler and Neirynck [16] will be adopted here.

Definition A.1 (Available Energy) Given an n-port N, let the available energy $E_{A, x}$ in state $x$ be the maximum energy that can be extracted from $\mathbf{N}$ when its initial state is $x$, with the convention that $E_{A_{x}}=+\infty$ if the available energy is unbounded. That is,

$$
E_{A, \Sigma}=\sup _{T} \int_{0}^{T}-u(t)^{*} y(t) d t
$$

Definition A.2 (Passivity) $N$ is passive if $E_{A x x}$ is finite for eact initial state $x$.

Note that this definition of passivity is directly tied to a statespace realization for the $n$-port in question. This is not objectionable for our purposes since we aim to draw conclusions for switching converters for which state models are readily obtained. In the context of a switching converter, the concept $0^{\text {? }}$ passivity is of use in viewing a controlled converter as an interconnection of various $n$-ports.

Incremental Passivity The definition given here follows the system theoretic framework of Desoer and Vidyasagar [20].

Definition A.3 (Energy in the Increment) Given an $n$ port $N$ with initial state $x$, let $\left(u_{1}(t), y_{1}(t)\right)$ and $\left(u_{2}(t), y_{2}(t)\right)$ be any two admissible input-output trajectories on $[0, T]$ with $T$ finite. The energy in the increment between the two trajectories is defined by

$$
W_{x}(T)=\int_{0}^{T}\left(u_{1}-u_{2}\right)^{*}\left(y_{1}-y_{2}\right) d t
$$

Definition A.4 (Incremental Passivity) An n-port $\mathbf{N}$ with irial state $x$ is incrementally passive at state $x$ if $W_{x}(T)$, the energy in the increment defined in (25), is nonnegative for every pair of admissible trajectories on $[0, T]$ with $T$ finite. If the network is incrementally passive at all states $x$ in the state space, it is said to be incrementally passive. The n-port is strictly incrementally passive at state $x$ if $W_{s}(T)>0$ whenever the two trajectories are distinct. The netwo-k is strictly incrementally passive if it is strictly increr entally passive at every state in the state-space.

Note $t$ at this definition is closely tied to the definition of passivity. A passive network can supply only finite energy while an inczementally passive network can absorb only nonnegative ersergy in the increment between two trajectories ( $W_{x}$ in (25)).
Relative Passivity Incremental passivity proves to be too strong a condition in the case of certain noulinear $n$-ports. In fact, many nonlinear networks that are not incrementally passive exhibit a closely related property that we shall term relative passivity. Another closely related notion, local passivity for a capacitor (or inductor) has been introduced in [16, 25]. However, our definition of relative passivity is potentially applicable to any type of network. To defiue a relatively passive network, we examine the energy in the increment with respect to a coustant nominal operating point.

Definition A.s (Relative Passivity) Given an n-port $\mathbf{N}$ with equilibrium state $x_{n}$ and nominal output $y_{n}$ corresponding to the constant input $u_{n}$, consider the admissible trajectory $(u(t), y(t))$ on $[0, T]$ that is obtained with initial state $x(0)=x_{n}$. The $n$-port is relatively passive at $x_{n}$ if

$$
W_{x_{n}}(T)=\int_{0}^{T}\left[u(t)-u_{n}\right]^{*}\left[y(t)-y_{n}\right] d t \geq 0
$$

for any finite $T$. The n-port is relatively passive if (26) holds for any nominal operating point. $\mathrm{N}$ is strictly relatively passive at $x_{n}$ if the inequality in (20) is strict whenever $x(T) \neq x_{m} . N$ is strictly relatively passive if it is strictly relatively passive for any constant nominal state.

In the case of lossless elements for which $H_{x_{n}}(T)$ is a function of only $x_{n}$ and $x(T), W_{x_{n}}(T)$ can be useful as a Lyapunov function.

\section{References}

[1] J. R. Wood, "Power Conversion in Electrical Networks," PhD Thesis, EECS Dept., MIIT, 1973.

[2] F. Harashima, H. Hashimoto, and S. Kondo, "MOSFET Converter-Fed Position Servo System with Sliding Mode Control," IEEE Power Electronics Specialists Conf. (PESC) Record, 1983, pp.73-79.

[3] R. Venkataramanan, A. Sabonovic, S. Cuk, "Sliding Mode Control of DC-to-DC Converters," Proc. 1985 Int. Conf. Indust. Elec. Contr. Instr. (IECON85), pp. 251-258.

[4] H. Sira-Ramirez, "Sliding Motions in Bilinear Switcled Networks," IEEE Trans. Circ. and Syst., vol. CAS-34, no. 8 , August 1987 , pp. 919-933.

[5] C. W. Deisch, "Simple Switching Control Method Changes Power Converter into a Current Source," IEEE PESC Record, 1978, pp. 300-306.

[6] A. Capel, G. Ferrante, D. O'Sullivan, and A. Weinberg, "Application of the Injerted Current Model for the Dynamic Analysis of Switching Regulators with the New Concept of $L C^{3}$ Modulator," IEEE PESC Record, 1978, pp. 135-147.

[7] A. Sabonovic, N. Sabonovic, and O. Music, "Slicling Mode Control of DC-AC Converters," IEEE PESC Record, 1986, pp. 560-566.

[8] T. Kailath, Linear Systems, Prentice-Hall, 1980. 
[9] W. Burns and T. Wilson, "Analytic Derivation and Evaluation of a State-Trajectory Control Law for DCDC Converters," IEEE PESC Record, 1977, pp.70-85.

[10] 5. Huffman, W. Burns, T. Wilson, and H. Owens, "Fast-Response Free-Running DC-DC Converter Employing a State-Trajectory Coutrol Law," IEEE PESC Record, 1977, pp.180-189.

[11] R. W. Erickson, S. Cuk, and R. D. Middlebrook, "Large-Signal Modelling and Analysis of Switching Regulators," IEEE PESC Record, 1982, pp. 240-250.

[12] R. W. Brockett and J. R. Wood, "Electrical Networks Containing Controlled Switches," Addendum to IEEE Symposium on Circuit Theory, April 1974.

[13] G. Salut, J.C. Marpinard, and M. Valentin, "Large Signal Feedback Control for Power Switching Conversion," IEEE PESC Record, 1985, pp. 741-750.

(14) F. Chen and X.S. Cai, "Design of Feedback Control Laws for Switching Regulators Based on the Bilinear Large Signal Model," IEEE PESC Record, 1989, pp. $468-475$.

[15] Oruganti, Yang, and Lee, "Implementation of Optimal Trajectory Control of Series Resonant Converter," IEEE PESC Record, 1987, pp. 451-459.

[16] M. Hasler and J. Neirynck, Nonlinear Circuits, Artech House, 1986.

[17] J.L. Wyatt, Jr., L.O. Chua, J.W. Gannett, I.C. Goknar, and D.N. Green, "Energy Concepts in the State-Space Theory of Nonlinear n-Ports: Part I-Passivity," IEEE Trans. Circ. and Syst., vol. CAS-28, no. 1, Jan. 1981.

[18] J.L. Wyatt, Jr., L.O. Chua, J.W. Gannett, I.C. Goknar, and D.N. Green, "Energy Concepts in the State-Space Theory of Nonlinear n-Ports: Part II-Losslessuess," IEEE Trans. Circ. and Syst., vol. CAS-29, no. 7, July 1982.

[19] J.L. Wyatt, Jr., "Lectures on Nonlinear Circuit Theory," VLSI Memo 84-158, revised August 1984, MIIT, Dept. EECS.

[20] C.A. Desoer and M. Vidyasagar, Feedback Systems: Input-Output Properties, Academic Press, 1975.

[21] S.R. Sanders, G.C. Verghese, and D. Cameron, "Nonlinear Control Laws for Switching Power Converters," Control - Theory and Advanced Technology, vol. 5, no. 4, December, 1989.

[22] 1. Horowitz, M. Sidi, and R. Erickson, "Quantative Feedback Synthesis for Non-Linear Switched-Mode Uncertain Regulators," Int. J. Electronics, vol. 57, no. 4, pp. $461-476,1984$.

[23] S.R. Sanders, "Nonlinear Control of Switching Power Converters," MIT Dept. EECS, 1989.

[24] S.R. Sanders and G.C. Verghese, "Synthesis of Averaged Circuit Models for Switched Power Converters," 1990 IEEE International Symposium on Circuits and Systems (ISCAS), New Orleans, LA, May 1990.
[25] L.O. Chua, Lecture Notes for Network Theory, EECS 223, University of California, Berkeley, 1980. 